\title{
Muon-decay medium-baseline neutrino beam facility
}

\author{
Jun Cao, ${ }^{1}$ Miao He, ${ }^{1}$ Zhi-Long Hou, ${ }^{1}$ Han-Tao Jing, ${ }^{1}$ Yu-Feng Li, ${ }^{1}$ Zhi-Hui Li, ${ }^{2}$ \\ Ying-Peng Song, ${ }^{3,1}$ Jing-Yu Tang, ${ }^{1, *}$ Yi-Fang Wang, ${ }^{1}$ Qian-Fan Wu, ${ }^{1}$ \\ Ye Yuan, ${ }^{1}$ and Yang-Heng Zheng ${ }^{4}$ \\ ${ }^{1}$ Institute of High Energy Physics, CAS, Beijing 100049, China \\ ${ }^{2}$ Sichuan University, Chengdu 610065, China \\ ${ }^{3}$ University of Science and Technology of China, Hefei, Anhui 230029, China \\ ${ }^{4}$ University of Chinese Academy of Sciences, Beijing 100049, China
}

(Received 11 February 2014; published 23 September 2014)

\begin{abstract}
Neutrino beam with about $300 \mathrm{MeV}$ in energy, high-flux and medium baseline is considered a rational choice for measuring CP violation before the more powerful Neutrino Factory is to be built. Following this concept, a unique neutrino beam facility based on muon-decayed neutrinos is proposed. The facility adopts a continuous-wave proton linac of $1.5 \mathrm{GeV}$ and $10 \mathrm{~mA}$ as the proton driver, which can deliver an extremely high beam power of $15 \mathrm{MW}$. Instead of pion-decayed neutrinos, unprecedentedly intense muon-decayed neutrinos are used for better background discrimination. The schematic design for the facility is presented here, including the proton driver, the assembly of a mercury-jet target and capture superconducting solenoids, a pion/muon beam transport line, a long muon decay channel of about $600 \mathrm{~m}$ and the detector concept. The physics prospects and the technical challenges are also discussed.
\end{abstract}

DOI: 10.1103/PhysRevSTAB.17.090101

\section{INTRODUCTION}

\section{A. Physics goals}

In the past two decades, neutrino physics has made tremendous advancements, with the discoveries of neutrino oscillations and measurements on the mass and mixing parameters [1]. In 2012, the last mixing angle $\theta_{13}$ was discovered to be nonzero at Daya Bay and other neutrino facilities [1-7]. The unexpected large $\theta_{13}$, about $9^{\circ}$, changes the scenario of the neutrino oscillation study. It becomes easier to measure the mass hierarchy (i.e., the sign of $\Delta \mathrm{m}_{31}^{2}$ ) and lepton $\mathrm{CP}$ violation phase. It is believed that the experiments to be carried out at the facilities either under construction or under planning, such as JUNO [8], PINGU [9], HyperK [10], LBNE [11], INO [12] and LBNO [13] in the coming decade can determine the neutrino mass hierarchy and measure the oscillation parameters very precisely. Thus, new experimental facilities now shall focus on the measurement of the $\mathrm{CP}$ phase. The Neutrino Factory (NF) [14] which was first proposed fifteen years ago and has been undergoing continuous design optimization [15] is considered to be the ultimate facility to measure all the neutrino oscillation parameters. However, it has many technical problems to be solved before it is materialized.

\footnotetext{
*Corresponding author. tangjy@ihep.ac.cn

Published by the American Physical Society under the terms of the Creative Commons Attribution 3.0 License. Further distribution of this work must maintain attribution to the author $(s)$ and the published article's title, journal citation, and DOI.
}

PACS numbers: 29.38.Db, 14.60.Pq, 29.20.Ej, 29.27.Eg

Other possible accelerator-based neutrino beam facilities for the CP phase, such as T2HK [16] and ESSnuSB [17-18], together with LBNE and LBNO, using neutrinos from pion decays instead of muon decays at the Neutrino Factory, suffer from background issues and possibly, insufficient beam power.

We propose here a dedicated facility, MOMENT (MuOn-decay MEdium baseline NeuTrino beam facility), for the $\mathrm{CP}$ phase measurement using neutrinos from muon decays. Some of the technically difficult issues at the Neutrino Factory, such as the muon cooling and muon acceleration, are avoided. Neutrinos are thus produced only at low energies, namely in the range of $200-300 \mathrm{MeV}$. This is feasible since the sensitivity to $\mathrm{CP}$ phase is independent to the neutrino energy and it is free from $\pi^{0}$ background which is shown as RES in Fig. 1.

In this paper, the design scheme for the MOMENT is presented.

\section{B. Facility concept}

To obtain a very intense neutrino flux for medium baseline neutrino oscillation experiments, a proton beam of very high beam power with an energy range of $1-3 \mathrm{GeV}$ is mandatory, at least at the level of a few megawatts. Both pulsed and continuous-wave (cw) proton beams can be considered. A common consensus in the international accelerator community is that the highest beam power can only be provided by a cw proton linac which can deliver a proton beam in tens of MW. However, there still exist no such proton linacs in the world due to extremely challenging technological difficulties in designing and 


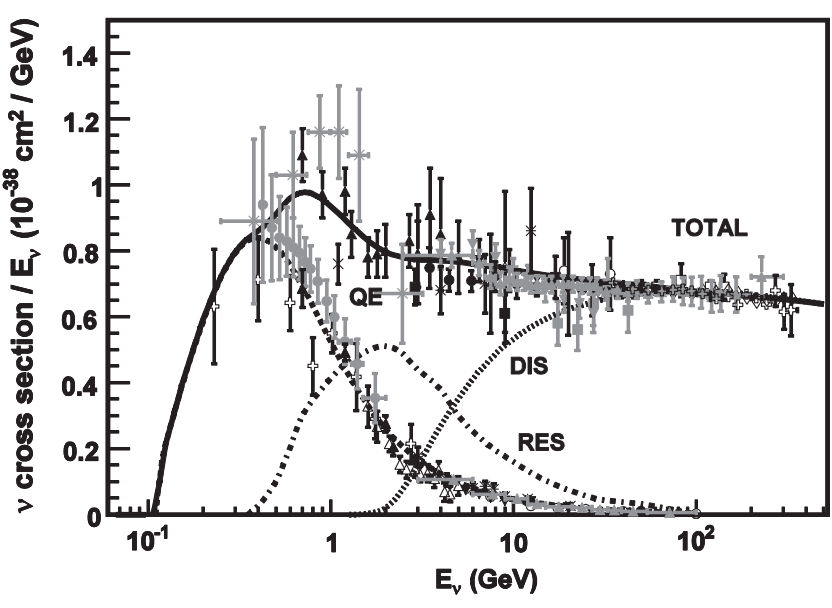

FIG. 1. Neutrino charge current cross sections as a function of neutrino energy [19-20]. RES: resonance-mediated, DIS: deep inelastic scattering, QE: quasielastic, and TOTAL: total cross section.

building them. Fortunately, China is among the first countries which started the development of such highpower proton linacs for nuclear waste transmutation. The China Accelerator-Driven System (China-ADS) project is developing a 15-MW superconducting linac in the $\mathrm{cw}$ mode in multiple stages [21]. Thus it is reasonable that we adopt a similar proton linac as the proton driver for MOMENT.

For pion production which is the first step for a neutrino beam, the target which should withstand the 15-MW beam power is a critical issue. The pion production target should be a radially thin one to facilitate the emission of pions from inside the target. By now, no solid target of such definition has proven that it can survive with the bombardment of a 15-MW proton beam [22]. Following the NF target design concept [15], we also adopt a mercury jet target. To collect pions efficiently, we should place the target in a very strong focusing field. With a cw proton beam, one cannot apply a focusing system based on magnetic horn as commonly used in many neutrino superbeams, since it can only work in short time duration and with a low repetition rate. Here the concept of placing the target in a high-field superconducting (abbreviated as SC) solenoid is adopted. The mercury jet target, the capture SC solenoid and the auxiliary devices constitute a very complicated target station. Compared with the pulsed proton beam at the NF, the $\mathrm{cw}$ proton beam at MOMENT helps a lot in easing heat mitigation and shock-wave problems in the target station [23], though the total heat deposit and the radiation dose rate due to the extremely high beam power are crucial problems to be solved here. As solenoid focusing does not distinguish between positive and negative pions which are produced simultaneously, the pion collection will include both types of pions. However, this will hinder the use of pion-decayed neutrinos for experiments, as the neutrinos generated by the opposite-charge pions will form unwanted background at the detector. The solution adopted here is to transport both types of pions and convert them into muons in a pion-decay channel, and then to separate the two muon beams in a special section.

Different from pion decays which happen mostly in a few tens meters, muons have much longer decay time. Therefore, the NF and the nuSTORM [24] have designed decay rings for muons to circulate many turns before they decay fully. However, with a $\mathrm{cw}$ proton beam as the primary one, the tertiary muon beam is almost a dc beam with a large momentum spread and transverse emittance. This hinders the storage of muons in a decay ring. Thanks to the relative low muon beam energy, we can design a long muon decay channel of about $600 \mathrm{~m}$ in which about $30 \%$ muons can decay, almost comparable to the decay efficiency in one long side of a racetrack decay ring. The selection of positive and negative muon beams is required before transporting the muon beam into the final decay channel. In addition, a bending section before the decay channel can help to get rid of the neutrinos by wrong charged muons and pions at the detector, to select the momentum range of the muon beam and to choose the direction of the neutrino beam.

The far detector is planned to be placed at a distance of approximately $100-150 \mathrm{~km}$, the baseline with maximum oscillation probability for the average neutrino energy. A near detector can measure the neutrino spectra and beam profile. The detection of muon decayed neutrinos is quite different from pion-decayed neutrinos. It is immune from the intrinsic background as long as the detector can distinguish the lepton charges [14]. The preselected momentum range for the muon beam can limit the maximum neutrino energy to about $400-500 \mathrm{MeV}$, and this is also helpful in reducing the $\pi^{0}$ background. For the neutrino beam from the decay of a muon beam, we have electron neutrinos and muon antineutrinos in the decay channel but all the four species $\left(\nu_{\mathrm{e}}\right.$, anti- $\nu_{\mathrm{e}}, \nu_{\mu}$, anti- $\left.\nu_{\mu}\right)$ at the far detector due to the oscillation. Therefore, we should design a detector capable of distinguishing the lepton charges, neutrino flavors and reject neutral current (NC) backgrounds from charge current (CC) interactions. Water Cherenkov (WC) detectors [25] have been proven to be effective in the flavor identification and $\mathrm{CC} / \mathrm{NC}$ discrimination. Moreover, the neutron tagging technique in gadolinium (Gd) doped WC detectors [26], which is still being developed [27] by the Super-Kamiokande collaboration, is superior to discriminate electron antineutrinos from electron neutrinos. Thus a large-volume Gd-doped WC detector may satisfy our requirement [28]. Of course, a magnetized sampling calorimeter can also satisfy the needs [15].

In summary, the proposed MOMENT facility mainly consists of a 15-MW superconducting linac in the cw mode as the proton driver, an assembly of the mercury jet target and the superconducting solenoids for pion production and 


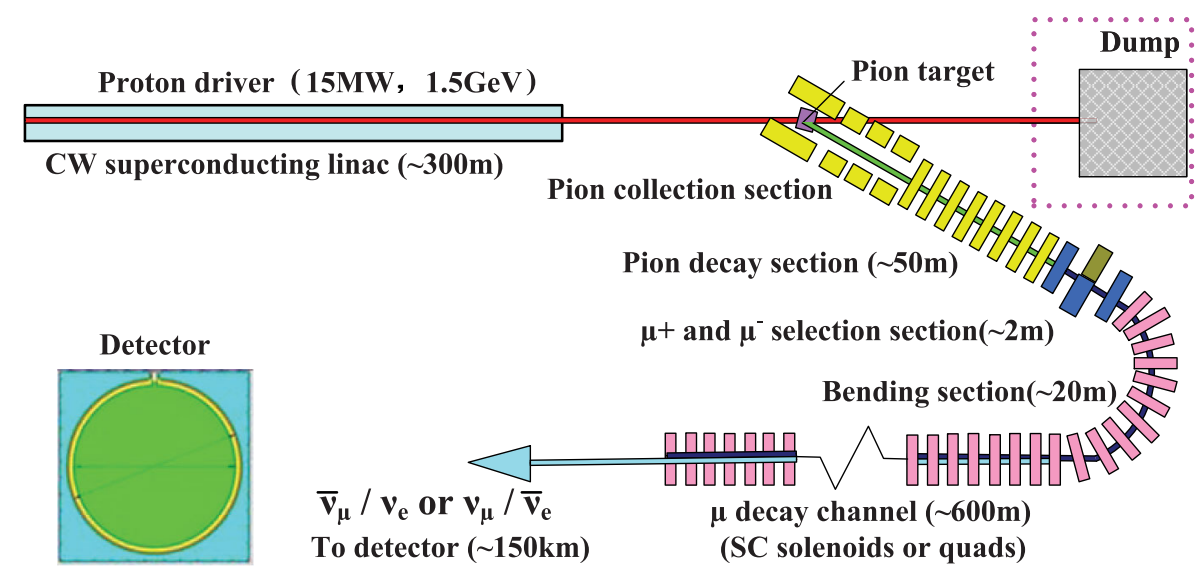

FIG. 2. Schematic layout of the MOMENT facility.

collection, a pion/muon beam transport line, a long muon decay channel of about $600 \mathrm{~m}$ and a possible large-size detector of Gd-doped water. The schematic layout is shown in Fig. 2. The neutrino source including the proton driver, target station and pion/muon transport channels can be hosted in the CSNS site [29], and a possible detector may be located at the JUNO site. The distance between the two sites is about $150 \mathrm{~km}$, consistent with the average neutrino energy of 200-300 MeV.

\section{PROTON DRIVER}

\section{A. Introduction}

The proton driver is a cw superconducting linac with a beam power of $15 \mathrm{MW}$, and the beam energy is still in optimization with a range of $1.5-2.5 \mathrm{GeV}$ depending on the efficiency of neutrino production and the cost. The nominal design energy is $1.5 \mathrm{GeV}$, the same as for the China-ADS linac [21], and this corresponds to a proton-on-target (POT) per year of $1.1 \times 10^{24} \mathrm{POT} / \mathrm{y}$. As there are a lot of technical challenges in building such a high beam power proton linac, we will profit from the China-ADS project and other $\mathrm{cw}$ linac projects which are developing the relevant techniques. For example, in about 2020, the China-ADS project is expected to complete an experimental facility with a linac of $250 \mathrm{MeV}$ working in the $\mathrm{cw}$ mode. All the SC cavity types needed for MOMENT except the high-beta one will have been tested with beam by then. As MOMENT is a scientific research facility, it is not necessary for the driver linac to have the extremely high reliability as required by ADS, and this will reduce the technical difficulties greatly and the cost significantly. We can simplify the design by taking away many installed redundancies.

\section{B. Lattice structure and beam dynamics simulation results}

The main difference between the MOMENT linac and the China-ADS linac are: the former has only one front end instead of two parallel ones for the latter; the former uses full applicable rf voltage as the nominal setting instead of about one-third reservation for the local compensation in the cases of cavity failures for the latter. This means that the MOMENT driver linac tolerates more frequent beam trips of longer duration than the China-ADS driver linac. The reliability of the MOMENT linac can be still guaranteed by applying the global compensation method as at SNS when some SC cavities fail [30]. The schematic layout of the MOMENT linac is shown in Fig. 3. In the following, some more details about different sections of the linac and beam dynamic studies are given.

The lattice structures for different sections are shown in Figs. 4 and 5. The front end is defined as the low-energy section including the ion source, LEBT, RFQ, MEBT, and Spoke012 section with the output energy of $10 \mathrm{MeV}$, which is totally the same as Injector Scheme I of the China-ADS linac. This is the most crucial part for a cw linac, where both the room-temperature RFQ, the very low-beta spoke cavities and the long cryomodule are difficult to develop. The test stand for the front end is under construction at IHEP.

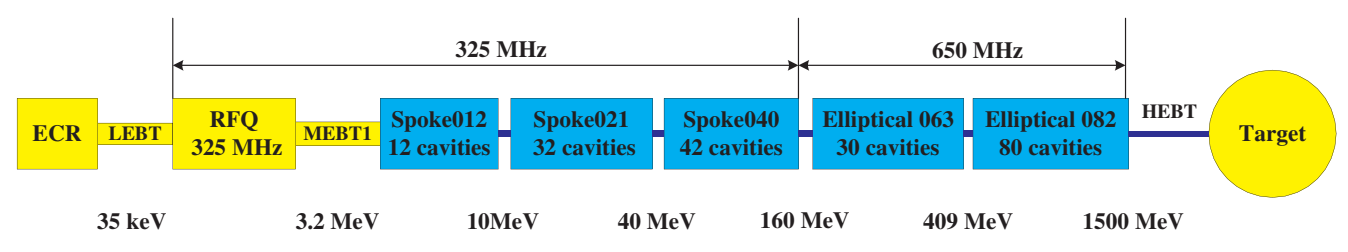

FIG. 3. Schematic layout of the MOMENT driver linac. 


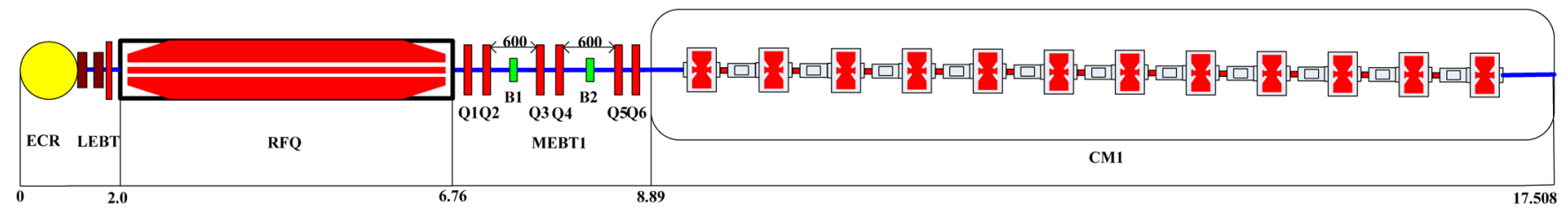

FIG. 4. Layout of the front end of the driver linac.

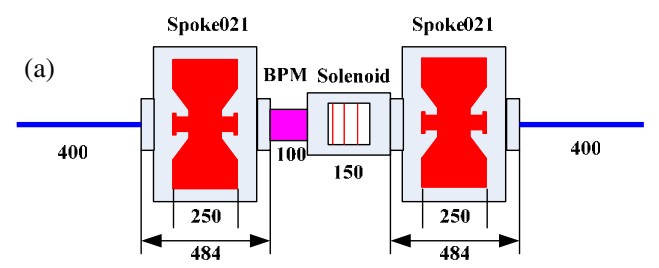

(b)
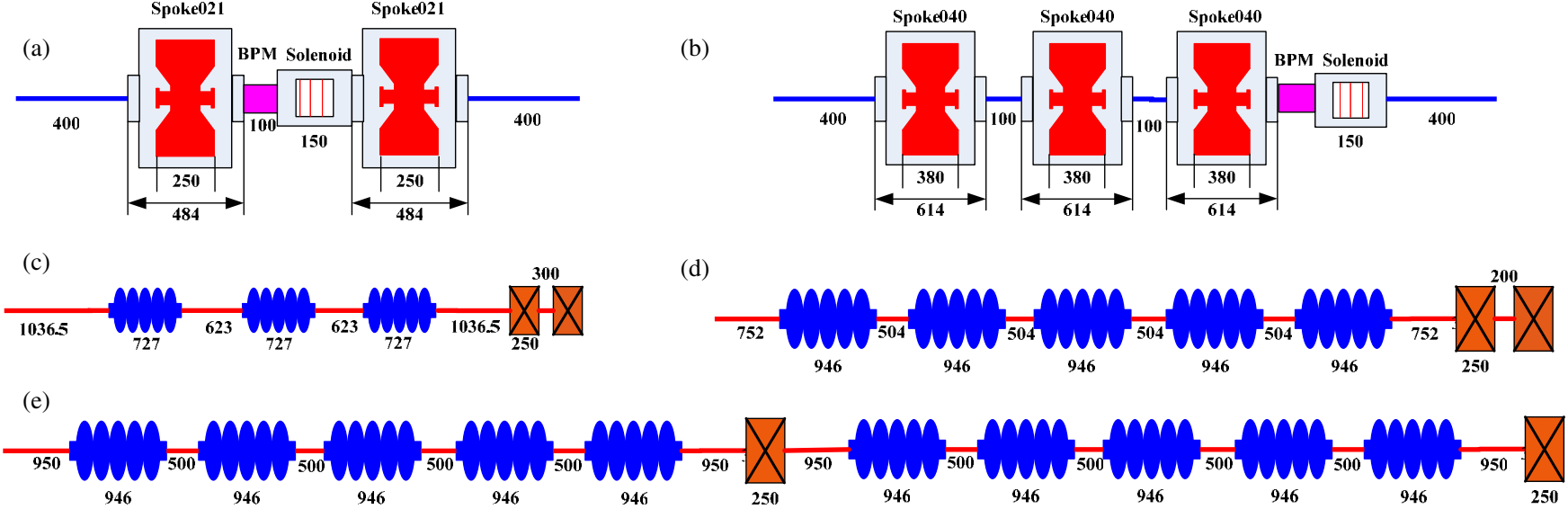

FIG. 5. Lattice structures of the main linac (a: Spoke021 section-RSR; b: Spoke040 section-R ${ }^{3}$ S; c: Ellip063 section- ${ }^{3}$ FD; d: Ellip082-R ${ }^{5} \mathrm{FD}$,; e: Ellip082-R $\mathrm{FR}^{5} \mathrm{D}$; S for SC solenoids, R for SC resonators, F/D for warm quadrupoles).

For the main linac part, there are five sections in four SC cavity types, and the sections are mainly determined by the focusing structures. As this is a high-power linac, the criteria of $1 \mathrm{~W} / \mathrm{m}$ beam loss rate along the accelerator [31-32] should be applied, and the preliminary study shows that it can be met with all errors included. The lattice structures should meet the requirements of the zerocurrent phase advance per period less than $90^{\circ}$ and smooth changes in the phase advance per meter between neighboring sections. The main parameters of the main linac are summarized in Table I.

The synchronous phase increases along the linac to obtain higher acceleration rate as the bunch length shrinks with the increasing energy, but it is important to have a large ratio of longitudinal acceptance to bunch area.

Multiparticle simulations have been performed to verify the validation of the design. The RFQ is simulated by PARMTEQM [33] and a truncated- $4 \sigma$ Gaussian distribution with 100000 particles with the TRACEWIN code [34] is used for the simulations of the rest of the linac. For all the SC cavities, the 3D field maps based on the cavity electromagnetic designs are used. For the transverse elements, the hard-edge approximation is used. Figures 6 and 7 show the rms envelopes and rms emittances along the linac. We can see that the envelopes change smoothly and the emittance growths are small.

\section{Alternative design}

As mentioned above, the proton energy may be optimized according to the global optimization between the neutrino production and the facility construction cost. Even for higher proton energy, the total beam power will be kept at $15 \mathrm{MW}$. This means that with higher energy the beam

TABLE I. Main parameters of the proton driver linac.

\begin{tabular}{|c|c|c|c|c|c|c|c|}
\hline & Spoke012 & Spoke021 & Spoke040 & Ellip063 & Ellip082-1 & Ellip082-2 & Total \\
\hline Energy (MeV) & 10 & 40 & 160 & 409 & 1000 & 1500 & 1500 \\
\hline Cavity number & 12 & 32 & 42 & 30 & 45 & 35 & 196 \\
\hline Focusing structure & $\mathrm{RS}$ & RSR & $\mathrm{R}^{3} \mathrm{~S}$ & $\mathrm{R}^{3} \mathrm{FD}$ & $\mathrm{R}^{5} \mathrm{FD}$ & $\mathrm{R}^{5} \mathrm{FR}^{5} \mathrm{D}$ & \\
\hline Total length (m) & 8.768 & 43.456 & 87.444 & 150.444 & 230.994 & 293.544 & 293.544 \\
\hline Section length (m) & 8.768 & 34.688 & 43.988 & 63.000 & 80.550 & 62.550 & \\
\hline Cryomodules & 1 & 8 & 7 & 10 & 9 & 7 & 42 \\
\hline Synchronous phase $\left(^{\circ}\right)$ & $-43--30$ & $-45--30$ & -25 & -20 & -15 & -15 & \\
\hline
\end{tabular}



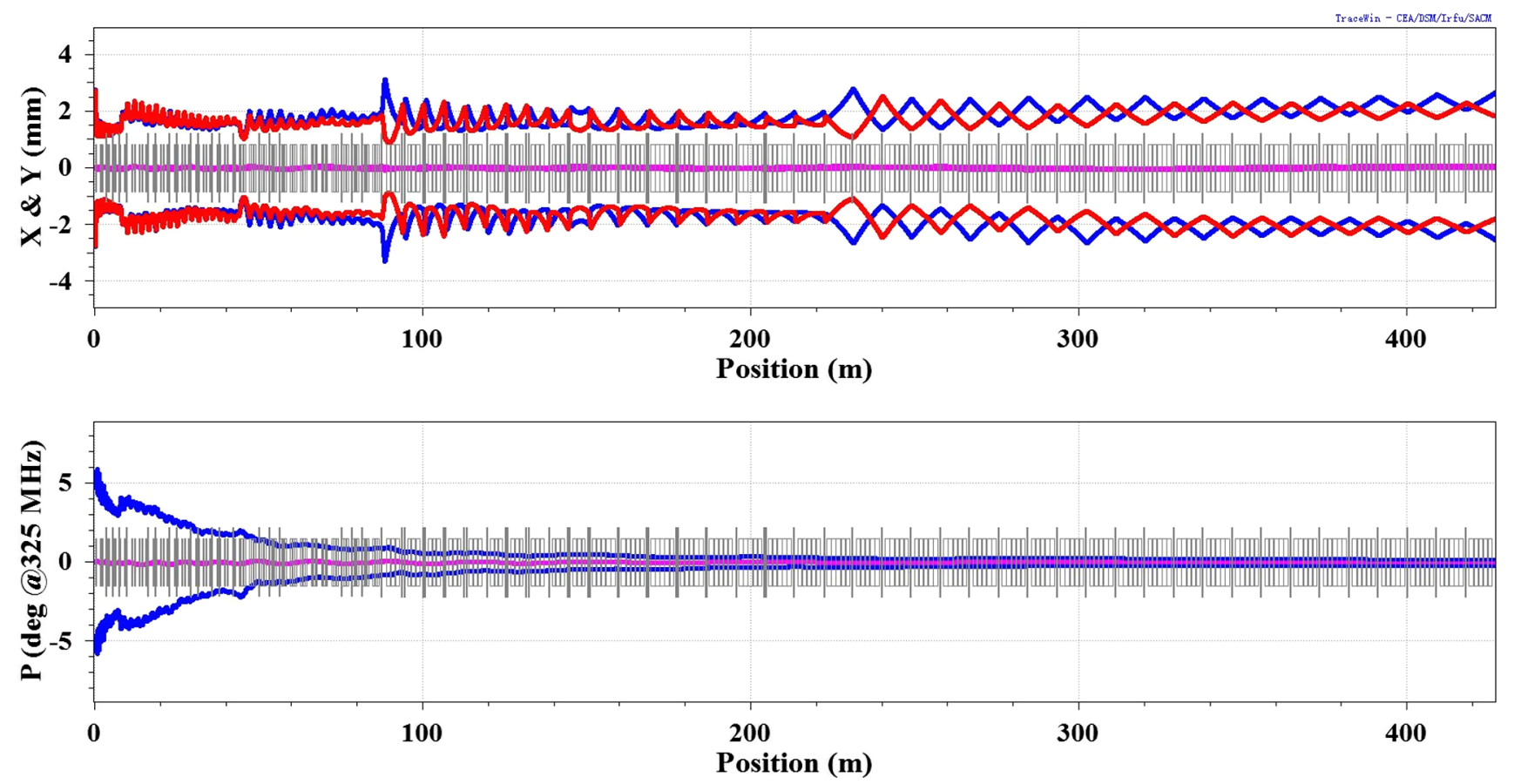

FIG. 6. The rms beam envelopes along the linac.

current will be lower which benefits the beam dynamics to some extent. The alternative design for extending the linac has been studied. A straightforward solution is just to add more Ellip082 cavities to cover the energy range from $1.5 \mathrm{GeV}$ to 2.0 or $2.5 \mathrm{GeV}$. Another solution is to use a new high-beta elliptical cavity type (Ellip093) to cover the highenergy part of 1.0 to $2.5 \mathrm{GeV}$. The study shows that with the latter one can save 15 cavities and the linac length of about 20 meters, which is considered only a modest gain that may not compensate for the additional effort on developing a new cavity type.

Following the progress of the China-ADS project, there will be more and more inputs from the hardware developments to be integrated in the design and the design will be further optimized.

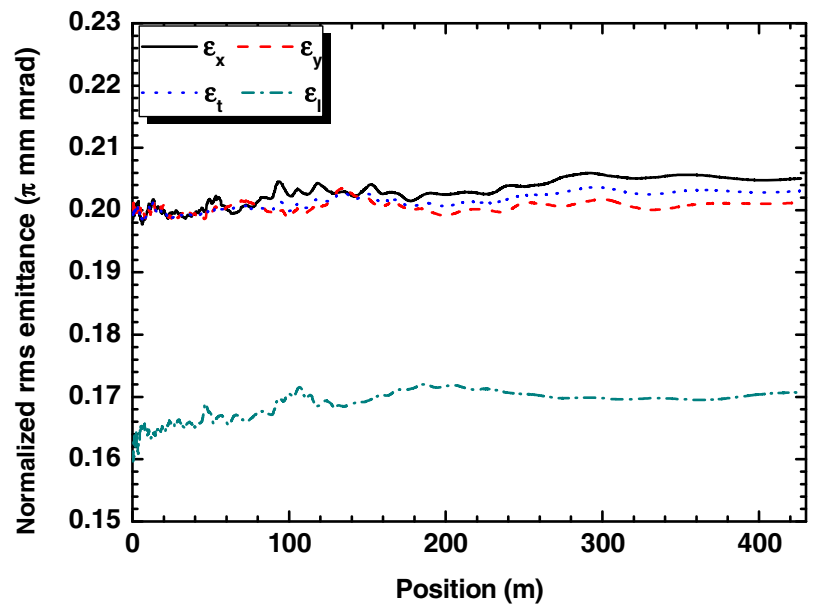

FIG. 7. Evolutions of the rms emittances along the linac.

\section{High-energy beam transport line}

The proton beam from the linac will be guided to impinge the target horizontally. Although this part can be flexibly designed to meet the general layout, an achromatic bending section is required to avoid the beam wobbling at the target and the back-streaming neutrons towards the linac tunnel. The final section will focus the proton onto a small spot of about 4-6 $\mathrm{mm}$ in diameter at the mercury jet target.

Although it is very difficult, we will continue the study to extract the used primary proton beam to an external beam dump instead of dumping it in the mercury pool as the IDS-NF does [15]. If it is possible to extract the spent proton beam from the target station, the dump beam line and the dump station are still technically difficult to build due to poor proton beam quality and very high beam power which is about a few megawatts.

\section{TARGET AND SECONDARY/TERTIARY PARTICLE COLLECTION}

\section{A. Mercury jet target}

As mentioned in Sec. IB, the pion production target should be a radially thin one to facilitate the emission from the target of the pions generated by the proton-target interaction and has to withstand the bombardment of a $15-\mathrm{MW}$ power proton beam. For now it is believed that a mercury jet target can meet the requirement, so it is adopted for the baseline design of the MOMENT target, just similar to the NF target design [35]. The MERIT collaboration has demonstrated by experiments that such a mercury jet target 
is technically feasible [36]. The studies also show that a very high field helps the confinement of the jet during the beam impinging period. Compared with the NF target or MERIT target, the peak beam power at MOMENT is much lower, so there will be no such problems as jet confinement and shockwave. Other options are also to be considered. For example, fluidized tungsten powder which is under study by RaDIATE [37], can be considered if the feasibility is demonstrated in the future.

\section{B. Pion production and collection}

Different proton beam energies from 1.5 to $2.5 \mathrm{GeV}$ have been used in the simulations to find a solution which is a trade-off among the technical difficulties, cost and neutrino production. At the moment, although the higher the proton beam energy the higher pion production yield, it is considered that the gain by increasing the proton beam energy is marginal if one keeps the beam power unchanged. The baseline design is with a $1.5-\mathrm{GeV}$ proton beam, but it can be modified later. For the production of negative pions, higher proton energy is also favored. The ratios between negative and positive pions at target are $0.49,0.63$ and 0.74 for $1.5,2.0$, and $2.5 \mathrm{GeV}$, respectively.

A high-field SC solenoid for pion capture was first proposed for a muon collider [38]. It can collect pions of both signs and perform adiabatic exchanges from the transverse momentum into the longitudinal momentum [39]. The experience of building such kind of pion capture system at MUSIC [40] and COMET [41-42] is used here in the design. The capture, decay and transport of secondary pions and tertiary muons are simulated mainly by using the G4BEAMLINE code [43]. It is found that the magnetic field level has a strong impact on the pion collection efficiency, especially on the core emittance of the pion beam. Different capture fields have been studied, and the field level of about $10 \mathrm{~T}$ or higher is considered necessary to efficiently capture the pions. However, the field level in this very large aperture magnet is limited by the superconducting magnet technology [44-45]. The baseline design adopts $14 \mathrm{~T}$ for the central field in the target region, similar to the design magnetic field by superconducting coils in the Neutrino Factory [15]. The use of a high-temperature superconducting magnet may permit operation at a higher field and will be studied in the future. Due to the production of very high neutron and gamma fluxes by the proton-target interaction, the heat deposit and irradiation effect in the SC coils are crucial problems for the cryogenic system and the quenching of superconductivity. A well-designed shielding to absorb the neutrons and gamma rays is mandatory, but it will increase the dimensions of the SC solenoid largely which has a very important impact on the magnetic field distribution and the cost. A sophisticated water cooling system is required to transfer the huge heat load of about $10 \mathrm{MW}$ in the shielding block. A brief summary of the power distribution in the target station is given in Table II.
TABLE II. Power distribution at the target station.

\begin{tabular}{lc}
\hline \hline & Power (MW) \\
\hline Pion beam into the decay channel & 0.7 \\
Spent proton beam & 2 \\
Proton beam along with pion beam & 0.3 \\
Deposit in the mercury jet & 2.5 \\
Deposit in the shielding block & 8.8 \\
Deposit in the SC solenoids & $<0.002$ \\
Backward shielding & 0.7 \\
\hline \hline
\end{tabular}

Figure 8 shows the schematic of the target station, which is somewhat similar to the NF target station [14].

The pions emitted from the target, together with the muons by either stopping pions or shortly decayed in-flight pions, are captured with a high efficiency. As low energy muons do not contribute to the neutrino oscillation experiment, they will be discarded in the downstream beam transport line. The thickness and length of the target are for the optimization of the pion production and capture. For the moment, the mercury jet target is designed to have a radius of $5 \mathrm{~mm}$ and an effective length of $300 \mathrm{~mm}$ which is defined as the intersection length between the mercury jet and beam. The proton beam spot is $1 \mathrm{~mm}$ in rms. The mercury jet has a small slanted angle with respect to the proton beam, which is to be optimized. The calculated total pion production rate is about 0.10 pion/proton in the intersection. With the capture field of $14 \mathrm{~T}$, the collection efficiencies of the forward pions/muons and total pions/ muons are about $82 \%$ and 58\%, respectively. A field tapering section by five SC solenoids is used to adiabatically decrease the field level from $14 \mathrm{~T}$ in the target region to 3-4 $\mathrm{T}$ in the decay/transport channels, as shown in Fig. 9. This process is important to convert the transverse momentum into the longitudinal momentum for better beam transport in the downstream beam transport lines. Figure 10 shows the momentum spectrum of the extracted pions. As a comparison, two other capture field levels of 7 and $10 \mathrm{~T}$ have also been studied.

\section{Heat deposit and radiation dose rate in the capture solenoid}

$\mathrm{Nb}_{3} \mathrm{Sn}$ superconducting coils [44] will be used for the capture solenoid. The main technological challenges are the high heat deposit and radiation dose rate in the SC coils by neutrons and gamma rays emitted from the target. A shielding structure by tungsten-carbide beads cooled by water [46-47] between the target and the SC coils is crucial in reducing the two parameters. FLUKA [48-49] simulations have been carried out to optimize the shielding design. The simulations show that the shielding can absorb almost all the gammas and the majority of neutrons. The heat deposit density is shown in Fig. 11. If we want to limit the energy deposited in the SC coils below $1 \mathrm{~kW}$ which is 


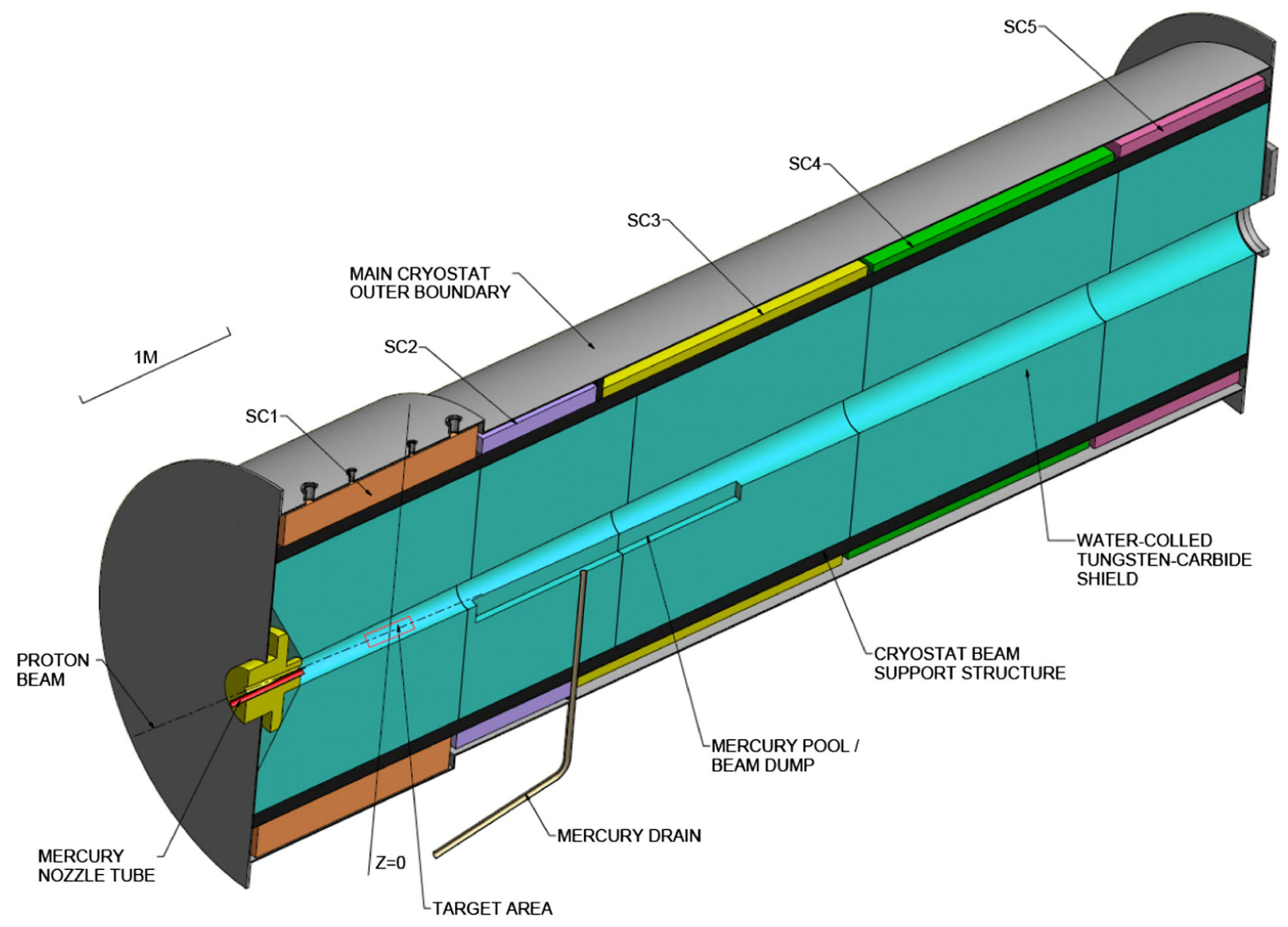

FIG. 8. Schematic of the MOMENT target station.

considered already a very heavy burden for the cryogenic system, the shielding thickness at the target location should be at least $800 \mathrm{~mm}$. This defines the inner radius of the SC coils as $1050 \mathrm{~mm}$. The inner bore of the shielding block is tapered to keep it away from the used protons and also the high-energy pions. The minimum inner radius of the shielding block at the target area is $200 \mathrm{~mm}$. With this configuration, the largest flux of neutrons with $E>0.1 \mathrm{MeV}$ escaping from the shielding inside the

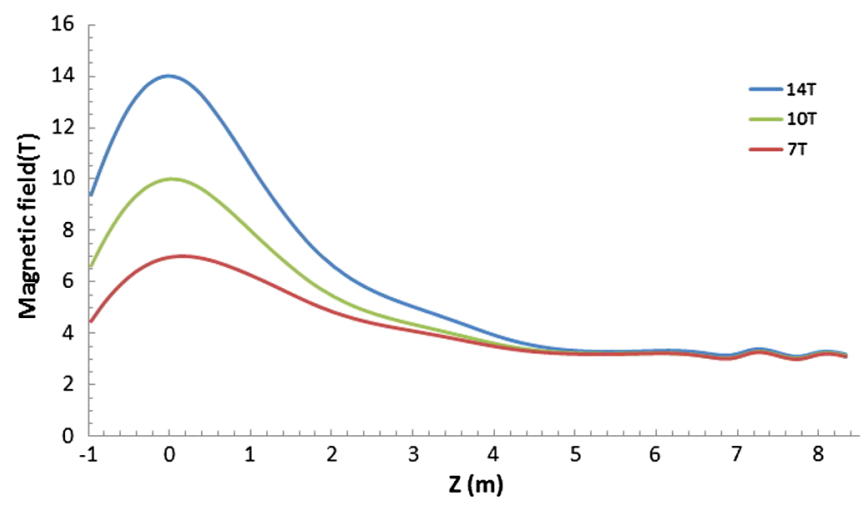

FIG. 9. Magnetic field pattern on axis along the field tapering section. solenoids is about $6 \times 10^{13} /\left(\mathrm{m}^{2} \mathrm{~s}\right)$, which means a fluence of $6 \times 10^{21} / \mathrm{m}^{2}$ for 10 years' operation with $10^{7}$ seconds per year. It is less than the case at ITER where the fluence of neutrons will be $10^{22} / \mathrm{m}^{2}$ [50]. There have been many investigations on the irradiation effect on superconductors and insulation materials using neutron sources. According to the studies [51], the superconductors by $\mathrm{Nb}_{3} \mathrm{Sn}$ are less

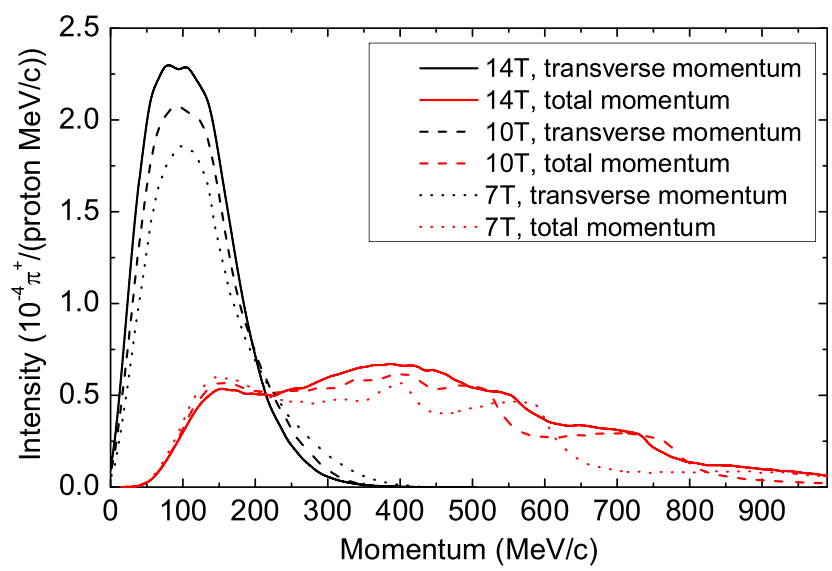

FIG. 10. Momentum spectrum of the extracted pions after field tapering with 7-T, 10-T and 14-T capture fields, respectively. 


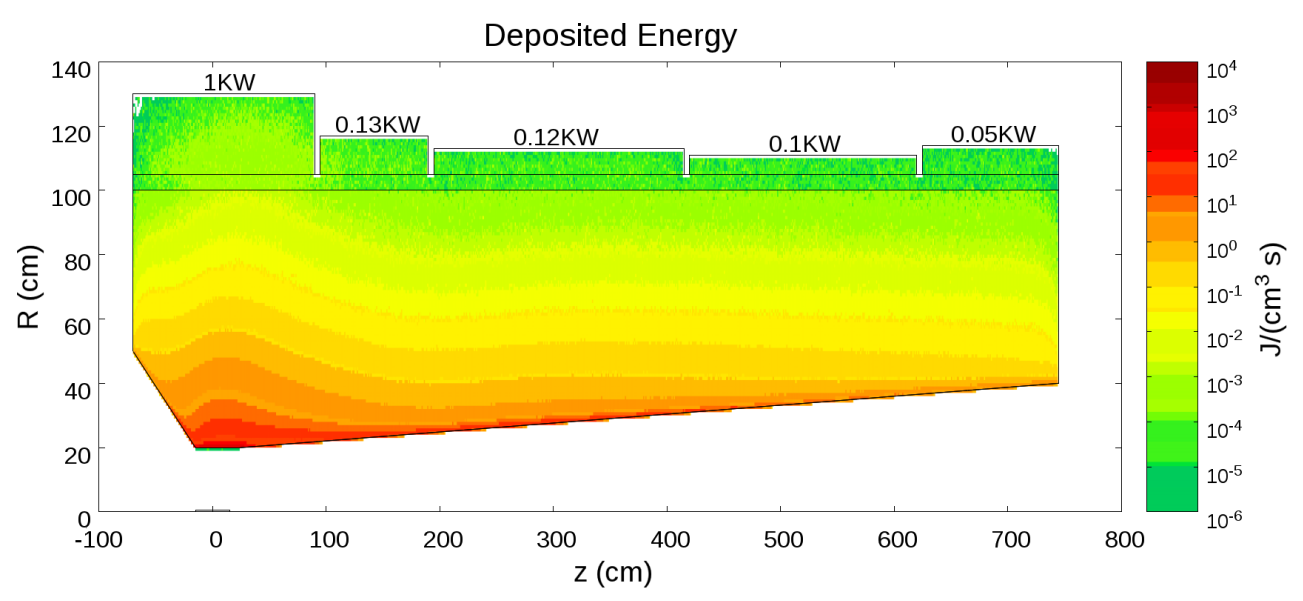

FIG. 11. Heat deposition in the shielding block and the SC coils.

concerned by neutron irradiation. For example, for a fluence of $1 \times 10^{22} \mathrm{n} / \mathrm{m}^{2}$ of neutron irradiation, the critical current of $\mathrm{Nb}_{3} \mathrm{Sn}$ was found even increased by $40 \%$ around $13 \mathrm{~T}$, though for a fluence over $10^{22} \mathrm{n} / \mathrm{m}^{2}$, the critical temperature (TC) degrades rapidly with increasing fluence. The main problem is with the electrical insulation of the magnet coil between turns and to ground. However, a more systematic study should be carried out in the future.

\section{Treatment of used protons}

Due to extremely high beam power, the proton beam poses a very challenging problem for the design of the target station. Only a small part of the primary beam power is converted into pion production and consumed by other nuclear reactions, and an important part is lost in the target by the ionization process. There will be two different proton compositions after the interaction between the primary beam and the target, one is the degraded primary proton beam which has lost a part of its energy or power mainly due to ionization, and the other is the scattered protons from the target during the interaction which spread over a large momentum range.

It will be very interesting to guide the degraded proton beam to an outer beam dump instead of sending it into the local mercury pool directly as default, so the neutron production yield in the target station can be largely reduced. This is important for designing the SC coils and the shielding block. However, the degraded beam quality and the sophisticated structure of the target station may hinder its feasibility in the engineering design. Higher proton energy and shorter intersection length will help retain a relatively better quality and higher beam power for the spent beam, which is relatively easier and more interesting to extract from the target station. An ongoing study is to find if there is a more-or-less realistic solution for the spent protons to pass laterally through the gaps of the SC solenoids which are arranged very closely.

The scattered protons will come out from the target area together with the pions. A part of them will be intercepted by an absorber, and others will go out into the downstream pion decay channel and heat up the SC solenoids and the beam pipes in the channel, which is to be discussed in Sec. IV.

In the future, much effort will be needed to study how to mitigate the beam power of the used protons.

\section{MUON BEAM TRANSPORT AND DECAY CHANNELS}

\section{A. Pion decay channel}

Thanks to the almost identical momentum for both a pion and its descendent muon, both pion and muon beams can be transported in the same focusing channel when pions continue to decay into muons [52]. In order to avoid the background by the neutrinos from pion decays at the detector, the muon beam line is designed to have a transport section where pions continue to decay, a bending section where very high-momentum pions are eliminated and a decay section where the properly selected muons decay to produce the required neutrino beam. Because our goal is to use neutrinos with the averaged energy close to $300 \mathrm{MeV}$, the required muon beam should have momentum close to $300 \mathrm{MeV} / c$. Therefore, the average momentum of $300 \mathrm{MeV} / c$ and the spread of about $\pm 50 \%$ are chosen for the muons in the decay section. This means that other pions or muons outside the momentum range which occupy more than $50 \%$ of the total collected pions from the target/ collection region, as shown in Fig. 12, should be eliminated by the bending section. The drop in the momentum spectrum is due to the stop band crossing for a certain energy which is caused by phase advance per period close to $\pi$ or $2 \pi$. We will try to reduce the beam loss due to this phenomenon by further optimization of the focusing channel. The pion/muon beam distributions in the transverse phase planes are shown in Fig. 13. Due to the cw proton beam and large momentum range, the time structure in the longitudinal phase plane blurs out along the pion decay channel. Due to very large transverse emittance and 


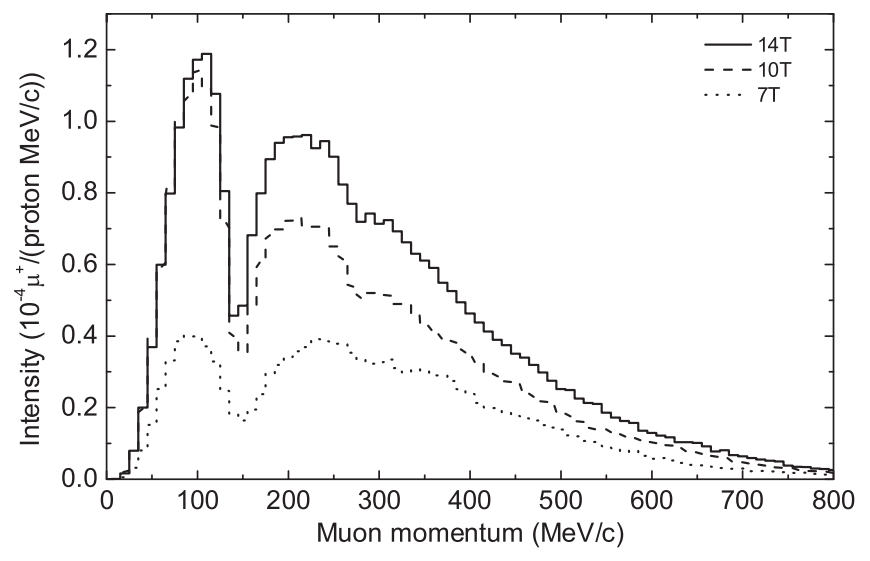

FIG. 12. Muon momentum spectrum at the end of the pion decay channel.

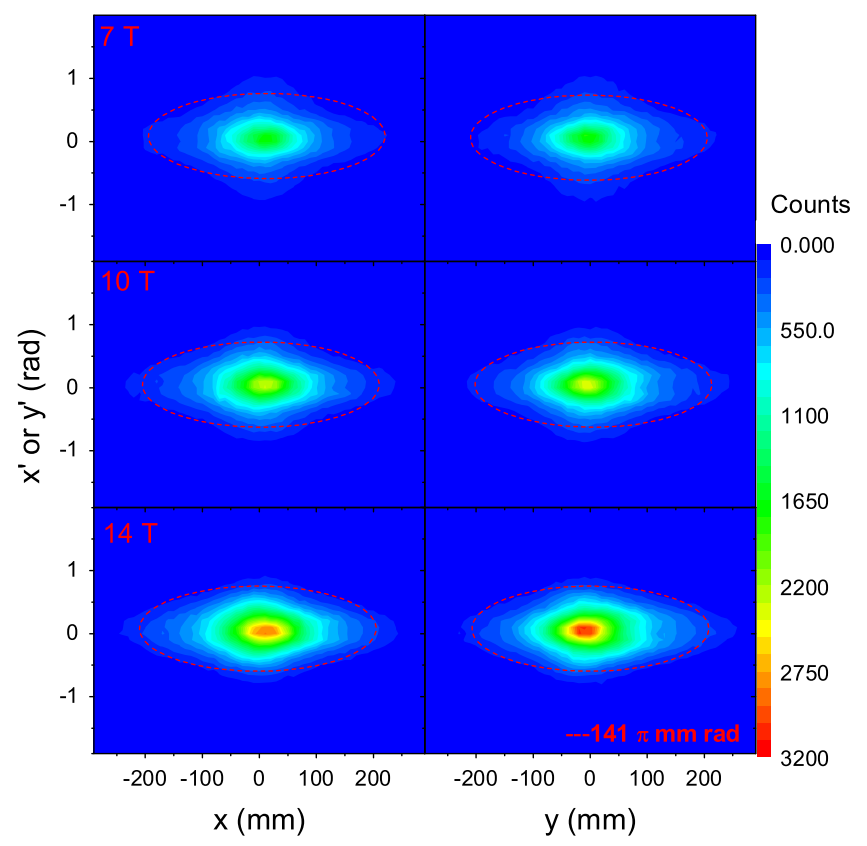

FIG. 13. Distributions of the pion/muon beam in the transverse phase planes at the end of the pion decay channel. The scale "Counts" used on the right is for relative comparison on density.

the $\mathrm{cw}$ beam, it looks impossible to apply a bunch rotation mechanism by rf cavities as in the case of the NF.

Studies show that with a very large transverse emittance, large momentum range and low average momentum, among different focusing structures SC solenoids are considered the best for the transverse focusing. The transverse acceptance and momentum acceptance along the pion/muon transport lines are very large in SC solenoidsbased focusing channels, and determined mainly by the requirement on the muon beam quality in the muon decay channel, which are in the orders of $100 \pi \mathrm{mm}$ rad and $\pm 50 \%$, respectively. Some details about the transport beam lines are shown in Table III. The transverse acceptance for a different momentum other than the reference momentum is inversely proportional to the momentum.

A pion decay channel with a length of about $50 \mathrm{~m}$ is designed to convert more than $90 \%$ pions into muons. Because there are also many scattered protons from the proton-target interaction coming out together with the pion/ muon beam, which possess high beam power, a welldesigned shielding is required to mitigate the heat to avoid the heat-up in the SC solenoids. A similar design such as the chicane type adopted by the NF can be applied here. In addition, the beam power related to the pion/muon beam also reaches up to about $100 \mathrm{~kW}$, which should be also treated carefully along the channel.

Except in the beginning part where the focusing strength is matched to the capture field in the target region and the curved solenoids are for eliminating protons, the long straight section of the channel is made of a pure Focusing-DriftFocusing-Drift focusing structure by SC solenoids. The averaged magnetic field for the SC solenoids is about $3.4 \mathrm{~T}$ and the aperture for the beam pipe is about $600 \mathrm{~mm}$.

\section{B. Selection of positive and negative muons}

Both positive and negative pions produced at the target are transported without preference by a solenoid-based focusing channel, and it is the same for the positive and negative muons. However, only one of the positive and negative muon beams is used for neutrino production at a time, so one of them should be removed from the main channel before entering the muon decay channel. Because the transverse emittance is very large here, it is difficult to use a simple chicane-type structure with usual dipole magnets. We have adopted a design using a group of superconducting dipole magnets which have also high field gradients to form a strong tripletlike focusing, which is still under optimization. The preliminary study shows that one needs magnets of about $3 \mathrm{~T}$ in field, $1 \mathrm{~T} / \mathrm{m}$ in field gradient and $600 \mathrm{~mm}$ in apertures. To increase the transmission efficiency of so large transverse emittance and momentum

TABLE III. Transverse acceptances of the pion/muon transport lines.

\begin{tabular}{lccccc}
\hline \hline & $\begin{array}{c}\text { Reference } \\
\text { momentum }(\mathrm{GeV} / \mathrm{c})\end{array}$ & $\begin{array}{c}\text { Aperture } \\
(\mathrm{mm})\end{array}$ & Peak field $(\mathrm{T})$ & $\begin{array}{c}\text { Acceptance } \\
(\pi \mathrm{mm} \text { rad })\end{array}$ & $\begin{array}{c}\text { Transmission } \\
\text { efficiency }\end{array}$ \\
\hline Pion decay channel & 320 & 600 & 3.7 & 141 & $68 \%$ \\
Bending section & 300 & 600 & 3.2 & 100 & $60 \%$ \\
Muon decay channel & 300 & 800 & 1.0 & 80 & $76 \%$ \\
\hline \hline
\end{tabular}




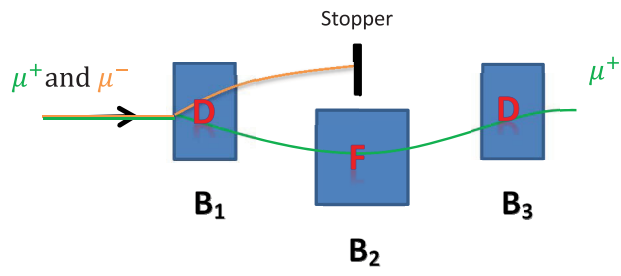

FIG. 14. Schematic of the $\mu^{+} / \mu^{-}$selection.

range, we are also considering adopting nonlinear Fixed Field Alternate Gradient synchrotron magnets instead of combined function dipoles. Figure 14 shows the schematic of the $\mu^{+} / \mu^{-}$selection. Together with the following bending section, this method can control the portion of the wrongcharge muons below $0.2 \%$. Other solutions such as the one by using bent solenoids [53] are also under investigation.

The discarded muon beam, either positive or negative, is so strong that it far outweighs the existing and proposed muon beams. It would be interesting to guide it to a dedicated muon facility for muon-based research, such as muon physics and $\mu \mathrm{SR}$ (muon spin rotation, relaxation and resonance) applications.

\section{Bending section}

As mentioned above, the bending section before the muon decay channel can help get rid of the pion-decayed neutrinos at the detector when needed and select the momentum range of the muon beam. This part can be flexibly adapted to the facility layout design with its bending angle and length. This conceptual design uses a bending angle of $90^{\circ}$ with a total length of $27 \mathrm{~m}$ and $45 \mathrm{SC}$ solenoids of $600 \mathrm{~mm}$ in diameter and $500 \mathrm{~mm}$ in length with a slanted angle of $2^{\circ}$, but the bending angle can be adjusted as needed for choosing the direction of the neutrino beam. The bending is made by slanted SC solenoids, which effectiveness has been proven at MuSIC [54-55]. The slanted angle for each solenoid has

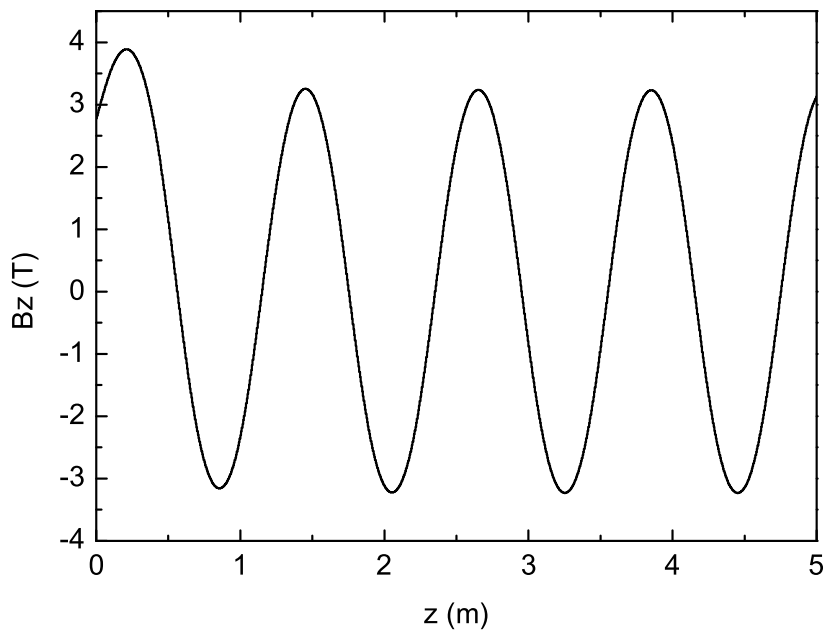

an important impact on the beam centroid excursion. We need many short solenoids with a small slanted angle such as $2^{\circ}$ used here. In order to reduce the coupling between the transverse phase planes, it is necessary to use alternate fields for the neighboring solenoids. Figure 15 shows the field distribution and the beam centroid excursions along the bending section. The oscillations on the vertical centroids are largely suppressed by the alternate fields. The large oscillation in the horizontal plane is due to the dispersion effect by bent solenoids, and the residual centroids at the exit can be largely suppressed by properly choosing the number of periods. We also need matching sections still by solenoids of different fields in the upstream and downstream channels for the matching in the transverse phase planes. The orbit errors in the entrance and exit of the bending section are corrected by using the correction coils attached to the matching solenoids and the beam position monitors.

There are some quite energetic pions which are still alive after passing through the pion decay section. They can continue to decay in the bending section. However, for those with high kinetic energy they may survive until the entrance of the downstream muon decay channel, and they produce neutrinos which may not be desired by the experiments. Therefore, it is important to limit the momentum acceptance of the bending section together to remove the high-energy pions. This can be assisted by applying a small dipole field to the solenoids by attaching special coils to the main coils. The dipole field is also helpful to reduce the beam centroid excursion or the aperture of the beam pipe which is strongly related to the cost of the channel.

\section{Muon decay channel}

The long decay channel of $600 \mathrm{~m}$ in length is to obtain a decay probability of about $33 \%$ for the average muon momentum of $300 \mathrm{MeV} / c$. Here the muon beam becomes a perfect dc beam, so is the neutrino beam. A matching

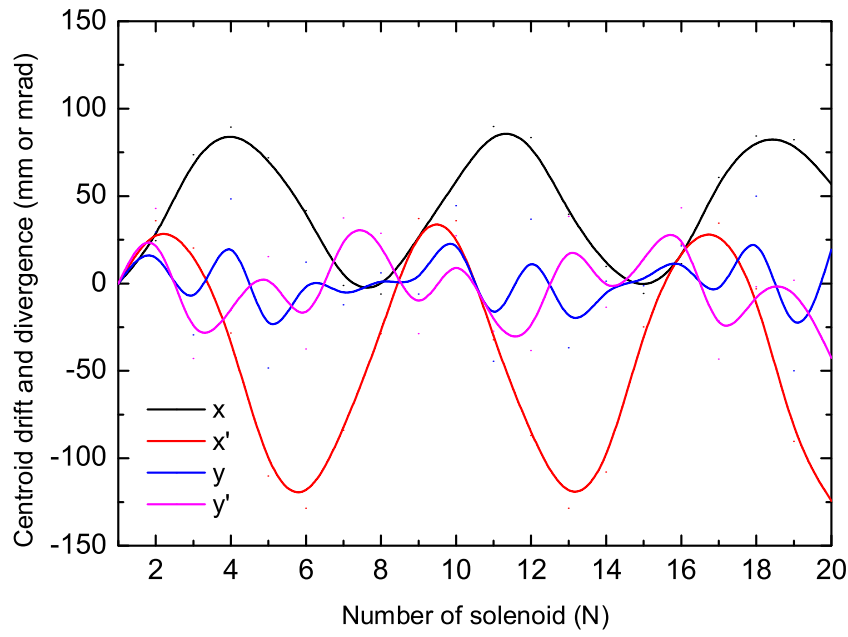

FIG. 15. Field distribution (left) and the beam centroid excursions (right) in a part of the bending section. 


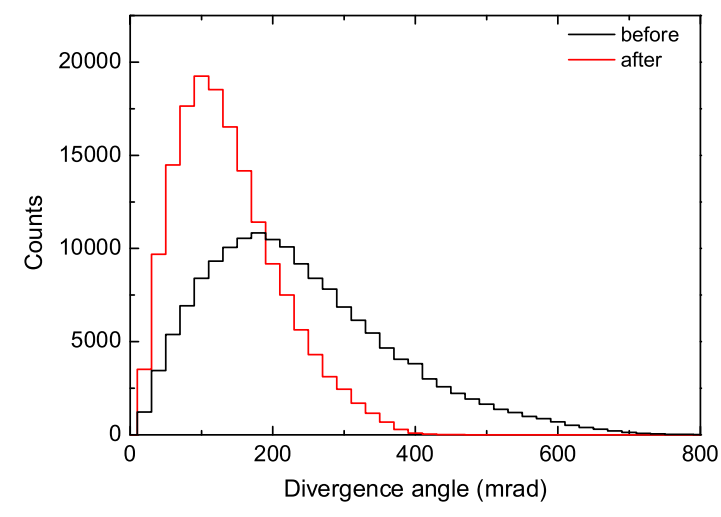

FIG. 16. Divergence angle distributions before and after the adiabatic matching section in the case of $14 \mathrm{~T}$ capture field.

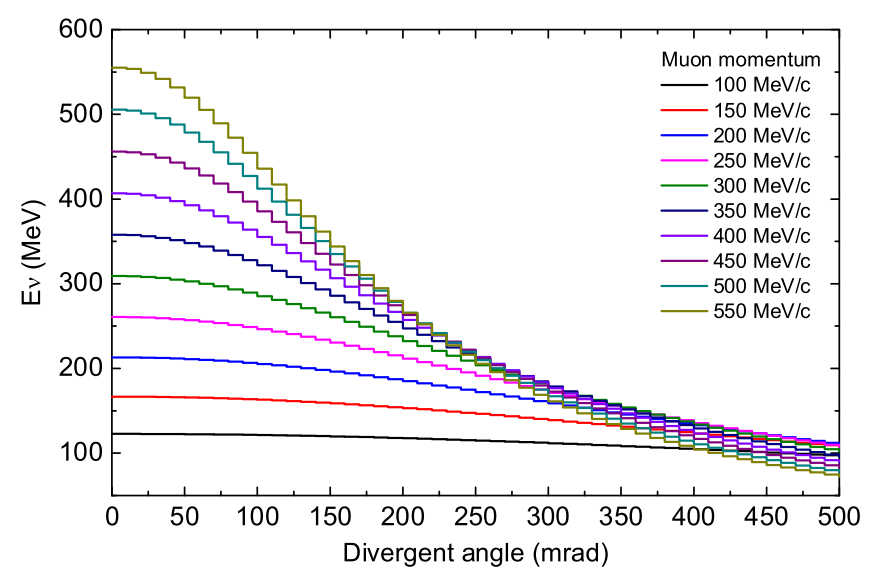

FIG. 17. Neutrino energy dependence on the divergent angle and momentum of a muon (this figure needs to be changed with "divergent angle").

section by decreasing the solenoid field from 3.7 to $1.0 \mathrm{~T}$ is to adiabatically convert partial transverse momentum to longitudinal momentum, which is similar to the matching section in the pion decay channel. The divergence angle

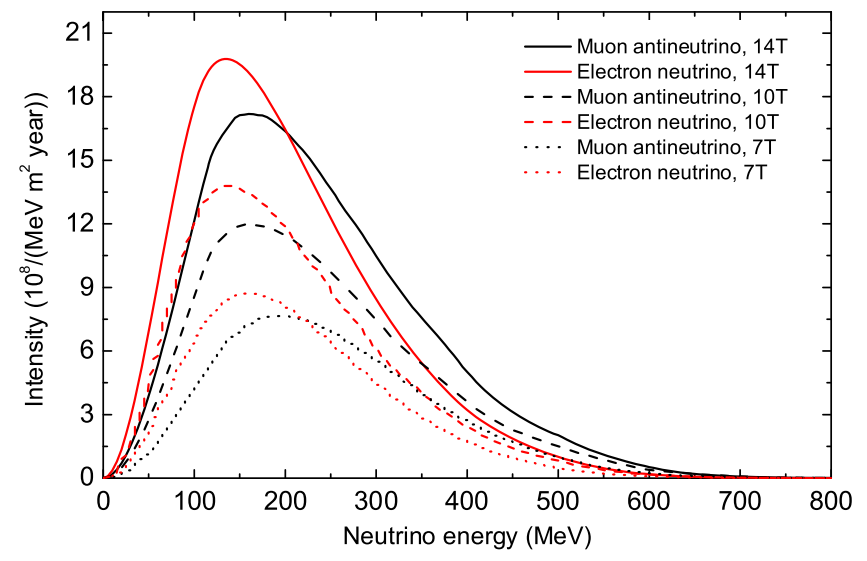

FIG. 18. Muon antineutrino energy spectra at the far detector of $150 \mathrm{~km}$.
TABLE IV. Neutrino fluxes at the far detector for different capture fields at the target.

\begin{tabular}{lc}
\hline \hline Field level & Neutrino flux $\left[\nu /\left(\mathrm{m}^{2} \mathrm{y}\right)\right]$ \\
\hline $7 \mathrm{~T}$ field & $2.1 \times 10^{11}$ \\
$10 \mathrm{~T}$ field & $3.3 \times 10^{11}$ \\
$14 \mathrm{~T}$ field & $4.7 \times 10^{11}$ \\
\hline \hline
\end{tabular}

distributions of the muon beam before and after the adiabatic section are shown in Fig. 16. This is very important to reduce the transverse divergent angle for the muon beam which is critical to obtain a required neutrino spectrum with an average energy larger than $200 \mathrm{MeV}$ at a far detector of $150 \mathrm{~km}$ in distance, because the high-energy neutrino is emitted only within a very small solid angle with respect to the muon's direction. Figure 17 shows the neutrino energy dependence on the divergent angle and momentum of a muon. The Focusing-Drift-Focusing-Drift focusing channel has a transverse acceptance of $65 \pi \mathrm{mm}$ rad for the reference momentum with a beam pipe of $800 \mathrm{~mm}$ in diameter. The total muon beam intensity in the decay channel is $1.0 \times 10^{15} \mu^{+} / \mathrm{s}$ or $1.8 \times 10^{22} \mu^{+} / \mathrm{y}$, and the neutrino yield (in pair) is $5.4 \times 10^{21} \nu / y$ which is more than twice the one at the Neutrino Factory. The neutrino spectra at the far detector with simulated particles are shown in Fig. 18, which does not take into account neutrino oscillations [56]. The averaged energy for muon antineutrinos is about $240 \mathrm{MeV}$, and the neutrino flux is $4.7 \times 10^{11} \nu /\left(\mathrm{m}^{2} \mathrm{y}\right)$ at the far detector which is lower than at the NF due to smaller $\gamma$ or lower energy. The neutrino fluxes for different capture fields are summarized in Table IV.

To limit the background by the decays of the pions which survive into the muon channel below $1 \%$ in the neutrino flux at the far detector, we can use methods either by the combination of the momentum selection in the chicane and the bending sections or by prolonging the pion decay channel from $50 \mathrm{~m}$ to about $100 \mathrm{~m}$ or by both methods.

Similar to the muon beam discarded by the muon beam selection section, the remaining undecayed muons at the end of the muon decay channel can also be used for other muon applications.

\section{DETECTOR CONCEPT}

A muon decay produces two neutrinos of different lepton charges and flavors, namely, a muon antineutrino and an electron neutrino for a $\mu^{+}$decay, a muon neutrino and an electron antineutrino for a $\mu^{-}$decay. Thus taking into account that partial neutrinos will change their flavors during the flight, all four neutrino flavors are present and the detector should be able to distinguish the charge and the flavor of neutrinos. It should also be able to distinguish charge current interactions (CC) from neutral current backgrounds, which could be very small in the case of low energy neutrino beams. 
As mentioned in Sec. I B, a Gd-doped WC detector may be suitable for our purpose [25]. From the experience of the Super-Kamiokande experiment, the water Cerenkov detector can well identify electrons from muons, and reject NC backgrounds from the CC signal. A Gd-doped WC detector can well identify electron antineutrinos by inverse beta decays (IBD) while electron neutrinos by the scattering type of signal. The magnetized iron neutrino detector or the magnetized liquid argon detector, which are being studied for the Neutrino Factory [14], is also well suitable. There is plenty of room for imagination in this direction, and novel detector concepts to match the special neutrino beam of MOMENT will be investigated. The target mass is expected to be submillion tons to quickly accumulate statistics.

The expected inclusive CC interactions of the appeared electron antineutrinos are around 1300 assuming $10 \mathrm{~T}$ field, 500 kton target mass, 10 years of operation when $\delta_{\mathrm{CP}}=0^{\circ}$, and about $30 \%$ of them are IBD interactions in the case of a Gd-doped WC detector. The backgrounds include the beam-unrelated ones (e.g., atmospheric neutrinos) and beam-related ones (e.g., the electron neutrino events scattered on oxygen with free neutron in the final state). As the exclusive CC interaction cross sections on these nucleons are still unclear in the literature, further studies on this aspect are required to estimate accurately these backgrounds.

For the dc neutrino beam, since the cross section of the neutrino interaction is extremely low, the inclusive event rate at the detector from the neutrino beam is several hundred per year per 500 kton including both $\mathrm{CC}$ and $\mathrm{NC}$ interactions. On the other hand, the window of the accidental event coincidence of a neutron capture is less than $1 \mathrm{~ms}$ even without Gd doped. Therefore, the event coincidence with a dc neutrino beam is negligible.

\section{DISCUSSION AND CONCLUSIONS}

Although the scheme shows no stop sign at the moment, there are many technical challenges to be solved by $R \& D$ efforts in the coming years and during the engineering construction. The most challenging one is with the proton driver and the target station. For the proton driver, there are issues like RFQ working in the cw operation mode, lowbeta superconducting cavities, high-power and large-scale rf amplifiers, cryomodules with many elements and high average heat load, very strict beam loss control, etc. For the target station, there are problems related to mercury jet and cooling circulation, high-field SC solenoids, shielding design, very high radiation dose rate and very high heat load in the solenoids, treatment of the primary and scattered proton beams, etc. The China-ADS project is executing a strong R\&D effort and will build experimental facilities to solve the design and technical issues related to a highpower $\mathrm{cw}$ proton linac. The neutrino beam facility will profit from its outputs. The target station and the pion decay channel at MOMENT share many technical issues with the
Neutrino Factory. A strong collaboration will be established between the two studies. Concerning the detector, which is still under the first investigation, the related technical issues are still to be identified.

The study on a muon-decayed medium-baseline neutrino beam facility presented above is still preliminary, more detailed studies and optimizations are to be carried out in the future. The extremely high neutrino flux and properly defined neutrino energy spectra make it very competitive in measuring the $\mathrm{CP}$ violation phase when other major neutrino oscillation parameters are determined. In addition, the facility provides an extremely intense muon beam of $10^{14} / \mathrm{s}$ in parallel to the neutrino beam, which can be exploited for muon physics or other researches based on muon sources. The principle of producing intense and lowenergy neutrino beam based muon decays is general and can be also applicable to other facilities.

Although the present scheme has attractive properties in the measurement of $\mathrm{CP}$ violation phase, the more powerful $\mathrm{NF}$ where it is driven by a relatively lower proton beam power and the tertiary muon beam is cooled in the phase spaces and accelerated to about $10 \mathrm{GeV}$ level, still has its importance in the particle physics. With a long baseline and neutrino beams produced by much better muon beam quality, the NF can perform very precise measurements for all the neutrino oscillation parameters. In the case that the $\mathrm{CP}$ violation is weak and very difficult to determine, the NF is still the final solution. Even when other experiments have found the $\mathrm{CP}$ violation phase, the more precise measurement at the NF is still helpful. In addition, the NF technology can be directly used for the muon collider which is the frontier machine for high-energy physics.

\section{ACKNOWLEDGMENTS}

The authors wish to express their thanks to the international neutrino beam community who welcomes the proposal and gives many valuable suggestions. The discussions within the Chinese high-energy physics community are also encouraging the preconceptual design. Thanks also go to Huayan He for making mechanical drawings. The study was supported jointly by National Natural Science Foundation of China (Projects No. 11235012, No. 11335009, and No. 10875099) and the CAS Strategic Priority Research Programs-JUNO and China-ADS.

[1] Y.F. Wang, in Proceedings of the 4th International Particle Accelerator Conference, IPAC-2013, Shanghai, China, 2013 (JACoW, Shanghai, China, 2013), p. 4010.

[2] F. P. An et al. (Daya Bay Collaboration), Phys. Rev. Lett. 108, 171803 (2012).

[3] F. P. An et al. (Daya Bay Collaboration), Chin. Phys. C 37, 011001 (2013). 
[4] Y. Abe et al. (Double Chooz Collaboration), Phys. Rev. Lett. 108, 131801 (2012).

[5] J. K. Ahn et al. (RENO Collaboration), Phys. Rev. Lett. 108, 191802 (2012).

[6] K. Abe et al. (T2K Collaboration), Phys. Rev. Lett. 107, 041801 (2011).

[7] P. Adamson et al. (MINOS Collaboration), Phys. Rev. Lett. 107, 181802 (2011).

[8] Y. F. Li, J. Cao, Y. F. Wang, and L. Zhan, Phys. Rev. D 88, 013008 (2013).

[9] D. J. Koskinen, Mod. Phys. Lett. A 26, 2899 (2011).

[10] K. Abe et al. (HyperK Collaboration), arXiv:1109.3262.

[11] C. Adams et al. (LBNE Collaboration), arXiv:1307.7335.

[12] N. K. Mondal, Eur. Phys. J. Plus 127, 106 (2012).

[13] S. K. Agarwalla et al. (LBNO Collaboration), arXiv: 1312.6520.

[14] S. Geer, Phys. Rev. D 57, 6989 (1998).

[15] R. J. Abrams et al. (IDS-NF Collaboration), Interim Design Report No. CERN-ATS-2011-216; arXiv:1112.2853.

[16] T. Ishida (Hyper-K Working Group), arXiv:1311.5287.

[17] E. Baussan et al. (ESSnu Collaboration), arXiv: $1309.7022 \mathrm{v} 3$.

[18] T. R. Edgecock et al., Phys. Rev. ST Accel. Beams 16, 021002 (2013).

[19] J. A. Formaggio and G. P. Zeller, Rev. Mod. Phys. 84, 1307 (2012).

[20] J. A. Formaggio and G. P. Zeller, arXiv:1305.7513.

[21] Z. Li et al., Phys. Rev. ST Accel. Beams 16, 080101 (2013).

[22] C. Densham, at the 2013 International Workshop on Neutrino Factories, Super Beams and Beta Beams, Beijing (2013).

[23] N. Simos, H. G. Kirk, P. Thieberger, H. Ludewig, J. O. Conor, L. Mausner, P.-T. Trung, K. T. McDonald, K. Yoshimura, and J. R. J. Bennett, J. Nucl. Mater. 377, 41 (2008).

[24] P. Kyberd et al., arXiv:1206.0294.

[25] S. Fukuda et al. (Super-Kamiokande Collaboration), Nucl. Instrum. Methods Phys. Res., Sect. A 501, 418 (2003).

[26] J. F. Beacom and M. R. Vagins, Phys. Rev. Lett. 93, 171101 (2004).

[27] H. Watanabe et al. (Super-Kamiokande Collaboration), Astropart. Phys. 31, 320 (2009); K. Abe et al. (T2K Collaboration), Phys. Rev. D 88, 032002 (2013).

[28] The detector concept and simulation results will be presented in a separate paper elsewhere.

[29] J. Wei, H. S. Chen, Y. W. Chen et al., Nucl. Instrum. Methods Phys. Res., Sect. A 600, 10 (2009).

[30] J. Galambos et al., in Proceedings of the Fifth International Workshop on the Utilisation and Reliability of High Power Proton Accelerators (Nuclear Energy Agency, Mol, Belgium, 2007).

[31] T. P. Wangler, Principles of RF Linear Accelerators (Wiley, New York, 1998).

[32] Proceedings of Workshop on Beam Halo and Scraping, Lake Como, , N. V. Mokhov and W. Chou (1999), http:// inspirehep.net/record/537420/files/?ln=EN.
[33] http://laacg1.lanl.gov/laacg/services/download_sf.phtml.

[34] http://laacg.lanl.gov/laacg/services/download_sf.phtml.

[35] http://slap.web.cern.ch/slap/NuFact/NuFact/NFNotes.html.

[36] K. T. McDonald et al., in Proceedings of the 23rd Particle Accelerator Conference, Vancouver, Canada, 2009 (IEEE, Piscataway, NJ, 2009), p. 795.

[37] http://www-radiate.fnal.gov/index.html.

[38] R. B. Palmer et al., in Proceedings of the 9th Advanced ICFA Beam Dynamics Workshop, edited by J. C. Gallardo, AIP Conf. Proc. No. 372 (AIP, New York, 1996), pp. 3-30.

[39] M. A. Green, in Proceedings of the 9th Advanced ICFA Beam Dynamics Workshop, edited by J. C. Gallardo, AIP Conf. Proc. No. 372 (AIP, New York, 1996), pp. 100-107.

[40] A. Sato et al., in Proceedings of 2nd International Particle Accelerator Conference (IPAC), San Sebastian, Spain, 2011, pp. 820-822.

[41] Y. Kuno, Nucl. Phys. B, Proc. Suppl. 225-227, 228 (2012).

[42] Y. G. Cui et al. (COMET Collaboration), Report No. KEK2009-10, 2009.

[43] T. J. Roberts et al., in Proceedings of the 24th Particle Accelerator Conference, PAC-2011, New York, 2011 (IEEE, New York, 2011), p. 373.

[44] A. V. Zlobin, IEEE/CSC \& ESAS European Superconductivity News Forum (ESNF), No. 16, 2011.

[45] P. Loveridge, in Solenoid Capture Workshop, 2010.

[46] N. Li et al., at the Third High-Power Targetry Workshop, 2007, Bad Zurzach, Switzerland.

[47] C. Song, P. Wang, and H. A. Makse, Nature (London) 453, 629 (2008).

[48] G. Battistoni et al., in Proceedings of the Hadronic Shower Simulation Workshop 2006, Fermilab, edited by M. Albrow and R. Raja, AIP Conf. Proc. No. 896 (AIP, New York, 2007), pp. 31-49.

[49] A. Ferrari, P. R. Sala, A. Fasso, and J. Ranft, Reports No. CERN-2005-10, No. INFN/TC_05/11, and No. SLAC-R-773, 2005.

[50] R. Fuger, M. Eisterer, F. Hengstberger, and H. W. Weber, Physica (Amsterdam) 468C, 1647 (2008).

[51] A. Nishimura, T. Takeuchi, S. Nishijima, G. Nishijima, T. Shikama, K. Ochiai, and N. Koizumi, Fusion Eng. Des. 84, 1425 (2009).

[52] E. B. Holzer, Reports No. CERN/PS 2001-059(PP) and No. CERN-NUFACT Note 092.

[53] R. B. Palmer and R. Fernow, in Proceedings of the 24th Particle Accelerator Conference, PAC-2011, New York, 2011 (Ref. [43]), p. 103.

[54] A. Sato, Y. Kuno, H. Sakamoto et al., in Proceedings of the 2nd International Particle Accelerator Conference, San Sebastián, Spain (EPS-AG, Spain, 2011), p. 820.

[55] M. Yoshida, M. Fukuda, K. Hatanaka, Y. Kuno, T. Ogitsu, A. Sato, and A. Yamamoto, IEEE Trans. Appl. Supercond. 21, 1752 (2011).

[56] J. Tang and W. Winter, Phys. Rev. D 80, 053001 (2009). 